\title{
A Method for Domain Name Data Acquisition Based on Web
}

\author{
Ye Jiajun ${ }^{1}$, Zhao Feng ${ }^{1+}$ \\ ${ }^{1}$ School of Computer Science, National University of Defense Technology, 58294 changsha, Hunan China
}

\begin{abstract}
The Domain Name System (DNS) is a vital element of the Internet infrastructure. And domain name data can be used as an essential data asset for some departments of a country or enterprises, for supporting reliable network service and ensuring network security. However, global domain name data acquisition is a very challenging problem. In this paper, we propose a method for domain name data acquisition based on Web, design and implement a domain name data acquisition system. We further conduct real experiments and evaluate the effectiveness of this method and the system. This method and the system provide supplements for the global domain name data acquisition techniques.
\end{abstract}

Keywords: domain name data acquisition, HTTP, WEB, active measurement.

\section{Introduction}

The Domain Name System (DNS) is a core service of the Internet, providing the mapping between human-readable names and IP addresses.

Domain Name data is an important resource that can be used as an essential data asset for some departments of a country or enterprises, playing an important role in ensuring network service and security. Each domain name is essentially a path of a large reverse tree called Domain Namespace which is huge and difficult to be enumerated: the DNS tree can have any number of branches on each node, with a depth of up to 127 layers. Each node has a text label with a maximum length of 63 characters. The entire structure is similar to the file system structure.

At present, The key domain name data are managed by Internet Digital Assignment Agency (IANA) of the US Department of Commerce's National Telecommunications and Information Administration (NTIA). Reference [1] suggests that the DNS root centralization implies vanishing and blinding risks, that is, vanishing refers to the risk of country domain names being blocked and blinding refers to the risk of being unable to access the Internet. Therefore, it is very important to obtain complete domain name data to to respond to these risks and other DNS problems. However, global DNS data are typically dispersed in authoritative nameservers, which usually close the name resource data transfer authority. Some domain name registration authority have some DNS data. But their domain name registration list are not allowed to be accessed without special permissions.In addition, huge domain namespace can not be enumerated.Thus how to obtain complete domain name data has become an important challenge.

Although there are some methods being proposed for obtaining domain name data,However, because of deployment restrictions and other factors, domain name data obtained are very limited.

Thus, We propose a method of domain name data acquisition based on Web. We design and implement a domain name data acquisition system. And we further conducts a series of experiments to evaluate their effectiveness and values..

\footnotetext{
+ Corresponding author.

E-mail address: fengzhao@nudt.edu.cn
} 
The rest of the paper is organized as follows. We present the existing methods in Section II. In Section III we describe the system design. Section IV describes the whole experiment and the results. Finally, we conclude the paper in Section V.

\section{Related Work}

Since DNS is one of the core components of the Internet, many institutions and researchers have conducted relevant researches on it. This paper focuses on the related background research in the areas of DNS measurement.

Currently, according to the existing research [2], the methods for obtaining domain name data include the following.

\subsection{Deploying the Traffic Measurment Device}

By deploying the traffic measurement device, the collected DNS message is analyzed to obtain the domain name and corresponding IP address. The amount of data that can be obtained by this method is closely related to the location where the traffic measurement equipment is deployed. However, it is powerless to the link that cannot be measured by the device (such as many foreign network links).

\subsection{Resolving the DNS Server Log}

At present, many Internet operators copy their designated DNS server logs to the analysis server for data mining. This method requires the collaboration of network operators or DNS server administrators. What's more, only a small number of DNS server logs can be obtained, while the rest are difficult to obtain.

\subsection{Discovering Based on Clawer}

Currently, the data collection modules of search engines have adopted the crawler method. In this method, some Portal Websites are selected as the search source to serve as the start of domain name crawling. The amount of data that can be obtained depends on a series of uncertain factors such as the quantity and quality of search sources. In addition, there is a problem of exponential explosion, and only a limited number of pages can be acquired under the limited time and bandwidth.

In the paper [3], researchers collected a unique long-term data set with daily DNS measurements for all the domains under the main top-level domains (TLDs) on the Internet (including .com, .net, and .org, comprising $50 \%$ of the global DNS name space). Futhermore, two case studies related to cloud e-mail services illustrate the value of measuring the DNS at this scale.

The work in [4] presented FluxBuster, a passive DNS traffic analysis system for detecting and tracking malicious flux networks. And the DNS traffic generated by local recursive DNS (RDNS) servers.

Florian Weimer described a approach to DNS replication in [5] called passive DNS replication. In the past, DNS replication was implemented based on zone file transfers, but this form required the cooperation of DNS administrators. To make matters worse, most name servers no longer allow zone file transfers. This approach concentrated on DNS response packets, and passively obtained DNS data through analysis and speculation.

\section{Method, System Design and Implement}

To avoid the large traffic overhead, difficult deployment and implementation, narrow coverage of existing DNS data acquisition methods .we propose a Web-based domain name data acquisition method, and provide a domain name data acquisition system based on Web.

The domain name data acquisition system consists of a web service detection module, a web page acquisition module, a page resolution domain name extraction module, and a domain name and IP mapping module. This system can be deployed on a server or host at any location. The structure between each module and database in the system is illustrated in Figure 1. 


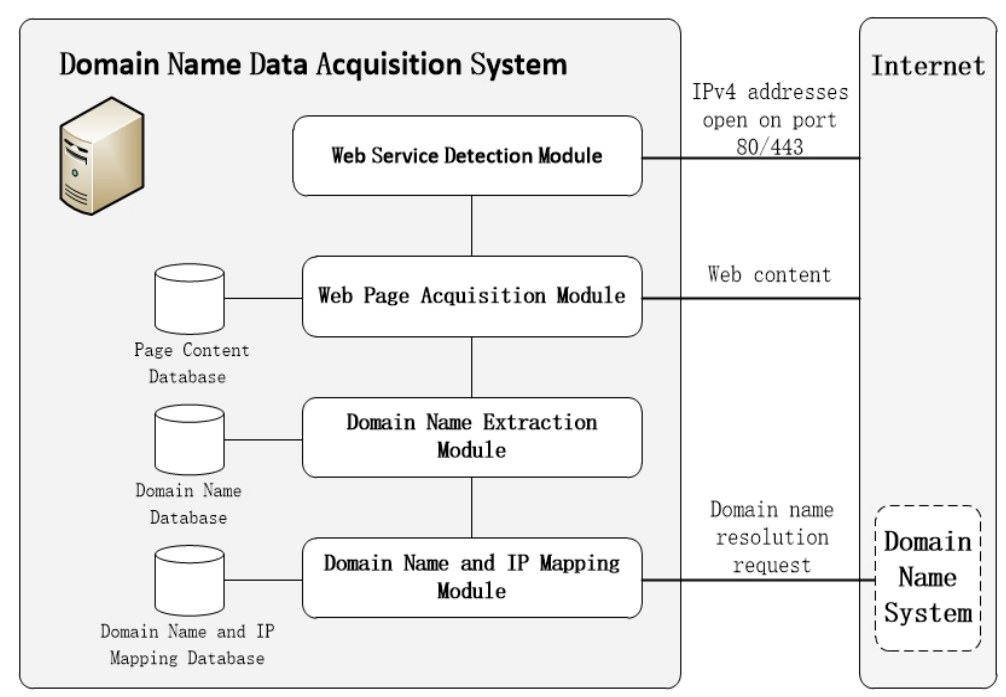

Fig. 1: The Architecture of the Domain Name Data Acquisition System

\subsection{Web Service Detection Module}

The Web services detection module scans the IPv4 unicast address space, obtaining the list of IPv4 addresses opening at port 80 and opening at port 443 in the Internet. This list will be sent to the Web page acquisition module. First of all, an appropriate scanning tool should be selected. Reference [6] introduces the functions of three popular network scanning tools, Nmap, Zmap and Masscan. After the test comparison and analysis for different types of scanning tools. Taking scanning speed and accuracy into consideration, we chose Masscan as the scanning tool. Then we set up exclusion address files and configuration files: private address network segment, reserved address network segment and multicast address network segment need to be excluded, and we specify the scan results include: IP address, port and flag which indicates whether the corresponding port is open and so on.

\subsection{Web Page Acqusition Module}

For every IPv4 address in the IPv4 address list open on port 80/443 or user-specified IPv4 address list, the Web page acquisition module sends HTTP/HTTPS Web page requests to the Internet concurrently. If the page content is received from the Internet, the Web page acquisition module constructs table items, including the corresponding IPv4 address, port number (80 or 443), returned page content, the HTTP Status Code, and stores the table items in the page content database.

This module is implemented by using Java programming and HttpClient as the main tool. HttpClient is used to provide an efficient, up-to-date, and feature-rich client programming toolkit that supports the HTTP protocol, and it supports the latest versions and recommendations of the HTTP protocol. In our impelmentation, we added HTTP request headers, which were a good way to disguise our program, making it more like browser access, thereby reducing the risk of request rejection.

\subsection{Domain Name Extraction Module}

The domain name extraction module selects all items in the database table whose HTTP status code is equal to 200, supposing there are $\mathrm{N}$ items that meet our requirements. We extract the links in the href attribute of the $<a>$ tag in each page. Then the domain names are extracted from the links by using the regular expression matching, and one link corresponds to extracting one domain name. And they are stored into the domain name database. The table entry of the domain name database contains three basic columns: IPv4 address, the port number corresponding to IPv4 address, and the list of domain names contained in the page corresponding to IPv4 address.

This process is implemented by using jsoup which is a Java HTML parser. Jsoup can parse a URL and HTML text directly with a very concise set of methods. By using the Jsoup tool, we can generate a Document object instance, generates a link element object instance Element, and obtains all the links on the page. Next, a regular expression is created to extract the host name from the link, and we compile it with the 
compile() method of the Pattern class, match the host name corresponding to the link with the matcher() method. A HashMap is created to hold key-value pairs of host names and occurrences (at least 1 time).

\subsection{Domain Name and IP Mapping Module}

First, we obtain $\mathrm{N}$ table items from the domain name database, and construct the non-repeating domain name set, so that assuming the number of elements in the set is N2. And then the domain name and IP mapping database is established by sending resolution requests to the domain name service system to resolve each domain name in the set.

In particular, this process is divided into the following steps

1) Initialize the variable $k=1$.

2) Take out the k-th domain name in the domain name set.

3) Initialize the k-th IP address set to be empty.

4) Send a DNS request to resolution server (such as the open domain name resolution server address 8.8.8.8 provided by Google).

5) The domain name and IP mapping module receives the domain name resolution response from the resolution server, and extracts the list of IP addresses corresponding to the domain name in the response (there may be multiple, may include IPv4 addresses or IPv6 addresses). If all the IP addresses in the IP list are already in the k-th IP address set, go to step 7, otherwise go to step 6.

6) The domain name and IP mapping module adds IP addresses in the IP list that are not in the k-th IP address set to the k-th IP address set, and go to step 4.

7) The domain name and IP mapping module composes the k-th domain name and the k-th IP address set into the k-th items of the domain-name and IP-address-set mapping database.

8) Let $k=k+1$, if $k \leqslant N 2$, go to step 2; if $k>N 2$, the domain name and corresponding IP address set of the global website are obtained, and the process ends.

\section{Experiments and Evalution}

To evaluate the effectiveness of the method and system, we query the IP adresses which belong to top $1,000,000$ Web sites as listed by Alexa in September,2018. Alexa is a website that publishes the world ranking of websites. It has a large number of urls and detailed ranking information.

In order to filter out the websites that can be accessed normally from the test location, we query the top one million websites one by one with domain names. Because of firewall and other factors, some websites are temporarily unavailable, so they will not be considered in this experiment. We recorded the results(mainly the returned HTTP status code) of these 1 million requests, and the result distribution is shown in Table 1.

As can be seen from Table 1, 55.04\% of all requests could returned the page correctly, while $38.37 \%$ of the status codes were unknown due to the above reasons. In the rest of the classification, and only status code 4XX accounted for $5.72 \%$, the rest are less than 1\%. Excluding the unknown returned results, $89.31 \%$ of the domain names can return to the page normally, which will be the main object of study as described below. Then we combine this part of the domain names into the set D1.

Table 1: Results of 1 Million Requests

\begin{tabular}{|l|l|l|l|}
\hline Type & Quantity & Propotion & Propotion(Exclude unknown) \\
\hline $2 X X$ & 550425 & $55.04 \%$ & $89.31 \%$ \\
\hline $3 X X$ & 941 & $0.09 \%$ & $0.15 \%$ \\
\hline $4 X X$ & 57171 & $5.72 \%$ & $9.28 \%$ \\
\hline $5 X X$ & 7746 & $0.77 \%$ & $1.26 \%$ \\
\hline $6 X X$ & 4 & Less than $0.001 \%$ & Less than $0.001 \%$ \\
\hline $7 X X$ & 2 & Less than $0.001 \%$ & Less than $0.001 \%$ \\
\hline
\end{tabular}




\begin{tabular}{|l|l|l|l|}
\hline Unknown & 383711 & $38.37 \%$ & \\
\hline Total & 1000000 & $100.00 \%$ & $100.00 \%$ \\
\hline
\end{tabular}

We used the DNS tool to query the IP of set D1, and save the domain name and IP address pair in set M1. In this experiment, we import the dnsjava-2.1.7 jar package and implement this process by Java programming. This is done for the scenario of a known IP address and an unknown domain name, and is a preparation for simulating this scenario. It should be noted that one domain name may correspond to multiple IP addresses. Upon completion of this task, we obtained 695,672 domain-ip address pairs from 550,425 domain names.

After completing this work, we input the 695,672 domain-ip addresses into the domain name data acquisition system for test and verification. Then unique entries were obtained, involving 49,798 IP addresses and 68,725 domain names.

Comparing the hostnames that appear most frequently on each page with the real domain names, the relevant combinations are divided into three categories.

Type A: the extracted hostname and domain name are exactly the same.

Type B: not exactly the same, but there is a subordinate relationship between the two names.

Type C: not exactly the same, and there is no hierarchy, but there is a complete overlap of characters.

In unique entries, there are 7,367 entries of Type A, 1,814 entries of Type B and 100 entries of Type C, accounting for $1.06 \%, 0.26 \%$, and $0.015 \%$ of the samples, respectively.

We can see that these results are not satistifying. Only a small proportion of domain names can be obtained based on IP address. In other words, a large proportion of web sites can not be accessed directly based on the IP addresses. The request for HTML page with IP address may be different from the normal return page, leading to the failure to get the correct page.We think there may be the following main reasons:

- Load balancing is a common means to improve the response speed of the server, alleviate the congestion problem and avoid various single point failures, so as to achieve high-quality network access effect. In this question, the website should avoid a large number of users accessing one or several servers that provide the same service at the same time. For this reason, many websites are prohibited from using IP address access directly, instead, load balancers implemented by software or hardware are used to distribute the work.

- Virtual host is a technology that can run multiple websites or services on a single host or host group. It is externally represented as multiple servers and they are completely independent of each other. The purpose is to make full use of hardware resources and avoid excessive address consumption. How to distinguish different virtual hosts with the same IP address becomes a new problem. The Host field in the HTTP request packet identifies the virtual host name and port number to solve the above problem. In this method, however, any host name hosted on a server cannot be known in advance, so the Host field can only be filled with the server's IP address by default, resulting in the server being unable to distinguish which virtual host the request specifically accessed. It can only return to the welcome page of the server software or hardware manufacturer or an error Bad Request with status code 400 .

- HTTPS certification. Many websites change to provide service based on HTTPS for security reason. The direct use of IP addresses cannot be verified by the domain name certificate of the HTTPS protocol of most websites. Only 7 pages are obtained based directionly on ip addresses by accessing port 443 in our experiments, so our method is only applicable to get domain name data by accessing port 80.

- Sometimes the client will send something that the server cannot handle, such as a malformed request message, or the most common is to request a non-existent URL. At this time, the server will return a 4XX status code. On the other hand, sometimes the server itself makes an error. And then the proxy will issue a 5XX HTTP status code to identify the details of the server error. 


\section{Conclusion and Future Work}

In this paper, we propose a Web-based method to obtain domain name data, design and implement a domain name data acquisition system,conduct real experiments and evaluate the effectiveness of this method and the system. And we find that a large proportion of web sites can not be accessed directly based on the IP addresses because of load balancing, virtual hosting, HTTPS certification and other various reasons. Still a proportion of domain names can be obtained based on our method and system, which can provide supplements for the global domain name data acquisition techniques.

Our experiments show that the web system in the Internet is a very complexy system, which will not work well without domain names. This suggests that new techniques should be explored for obtaining global domain name data.

\section{Acknowledgment}

This work is supported by Tianhe Supercomputer Project 2018YFB0204301 and National Key R\&D Program of China, 2018YFB1800205.

\section{References}

[1] Fang Binxing. Discussing Autonomous Root Domain Name Resolution System Based on National Alliance from "National Network Sovereignty"[J]. Information Security and Communication Confidentiality, 2014(12):34-37.

[2] Sun Tao. Research and implementation of domain name discovery analysis based on crawlers[D].

[3] Rijswijk-Deij R V , Jonker M , Sperotto A , et al. A High-Performance, Scalable Infrastructure for Large-Scale Active DNS Measurements[J]. IEEE Journal on Selected Areas in Communications, 2016, 34(6):1877-1888.

[4] Perdisci R , Corona I , Giacinto G . Early Detection of Malicious Flux Networks via Large-Scale Passive DNS Traffic Analysis[J]. IEEE TRANSACTIONS ON DEPENDABLE AND SECURE COMPUTING, 2012, 9(5).

[5] F. Weimer, "Passive DNS replication,” in Proc. 17th Forum Incident Response Secur. Teams Conf. (FIRST), 2005.

[6] Zhang Xingliang, Zhang Hongbo. Comparison and Analysis of Network Security Scanning Tools[J].Computer Knowledge and Technology, 2018, v.14(11):62-63.

[7] Zhang Weiwei, Gong Jian, Liu Shangdong, et al. DNS traffic monitoring for backbone networks[J]. Journal of Software, 2017(9).

[8] Tian Shiqi. Implementation and Traffic Analysis of DNS Traffic Collection System[D]. 2017.

[9] Shengyuan Wang. Research on Active Measurement of DNS Infrastructure Behavior and Performance[D].

[10] Shi Yonghui. Design and Implementation of DNS Server Automatic Discovery and Tracking System[D].

[11] De Vries W B , Van Rijswijk-Deij R , De Boer P T , et al. [IEEE 2018 Network Traffic Measurement and Analysis Conference (TMA) - Vienna, Austria (2018.6.26-2018.6.29)] 2018 Network Traffic Measurement and Analysis Conference (TMA) - Passive Observations of a Large DNS Service: 2.5 Years in the Life of Google[C]// 2018:1-8. 